NORDITA-2000/65 HE

\title{
Non-Abelian Stokes Theorems in Yang-Mills and Gravity Theories
}

\author{
Dmitri Diakonov ${ }^{*}$ and Victor Petrov* \\ • NORDITA, Blegdamsvej 17, DK-2100 Copenhagen Ø, Denmark \\ * St.Petersburg Nuclear Physics Institute, Gatchina 188 350, Russia \\ E-mail: diakonov@nordita.dk, victorp@thd.pnpi.spb.ru
}

\begin{abstract}
We recall the non-Abelian Stokes theorem for the Wilson loop in the Yang -Mills theory and discuss its meaning. Then we move to 'gravitational Wilson loops', i.e. to holonomies in curved $d=2,3,4$ spaces and derive 'non-Abelian Stokes theorems' for these quantities as well, which are similar to our formula in the Yang-Mills theory. In particular we derive an elegant formula for the holonomy in the case of a constantcurvature background in three dimensions and a formula for small-area loops in any number of dimensions.
\end{abstract}

\section{Introduction}

One of the main objects in the Yang-Mills theory as well as in gravity is the parallel transporter along closed contours, or holonomy. In Yang-Mills theory it is conventionally called the Wilson loop; it can be written as a path-ordered exponent,

$$
W_{r}=\frac{1}{d(r)} \operatorname{Tr} \mathrm{P} \exp i \oint d \tau \frac{d x^{\mu}}{d \tau} A_{\mu}^{a} T^{a},
$$

where $x^{\mu}(\tau)$ with $0 \leq \tau \leq 1$ parametrizes the closed contour, $A_{\mu}^{a}$ is the Yang-Mills field (or connection) and $T^{a}$ are the generators of the gauge group in a given representation $r$ whose dimension is $d(r)$. In curved Riemannian spaces the 'gravitational Wilson loop' or holonomy 
for $d$-dimensional vectors can be also written as a trace of the path-ordered exponent of the connection, this time of the Christoffel symbol,

$$
W_{\text {vector }}^{G}=\frac{1}{d}\left[\mathrm{P} \exp -\oint d \tau \frac{d x^{\mu}}{d \tau} \Gamma_{\mu}\right]_{\kappa}^{\kappa} .
$$

One can also consider parallel transporters of spinors in curved background: in this case the holonomy is defined not by the Christoffel symbols but by the spin connection which is not uniquely determined by the metric tensor, see the precise definitions below.

The Yang-Mills Wilson loop is invariant under gauge transformations of the background field $A_{\mu}$; the gravitational Wilson loop is invariant under general coordinate transformations or diffeomorphisms, provided one transforms the contour as well.

It is generally believed that in three and four dimensions the average of the Wilson loop in a pure Yang-Mills quantum theory exhibits an area behaviour for large and simple contours (like flat rectangular). This should be true not for all representations but those with ' $N$-ality' nonequal zero; in the simplest case of the $S U(2)$ gauge group these are representations with half-integer spin $J$.

One of the difficulties in proving the asymptotic area law for the Wilson loop in halfinteger representations (and proving that in integer representations it is absent) is that the Wilson loop is a complicated object by itself: it is impossible to calculate it analytically in a general non-Abelian background field. Meanwhile, it is sometimes easier to average a quantity over an ensemble than to calculate it for a specific representative. However, in case of the Wilson loop the path-ordering is a serious obstacle on that way.

A decade ago we have suggested a formula for the Wilson loop in a given background belonging to any gauge group and any representation [1]. In this formula the path ordering along the loop is removed, but at the price of an additional integration over all gauge transformations of the given non-Abelian background field, or, more precisely, over a coset depending on the particular representation in which the Wilson loop is considered. Furthermore, the Wilson loop can be presented in a form of a surface integral [2], see the next section. We call this representation the non-Abelian Stokes theorem. It is quite different from previous interesting statements [3, 4, 5, 6] also called by their authors "non-Abelian Stokes theorem' but which involve surface ordering. Our formula has no surface ordering. A classification of 'non-Abelian Stokes theorems' for arbitrary groups and their representations has been given recently by Kondo et al. [7] who used the naturally arising techniques of flag manifolds.

Though these formulae usually do not facilitate finding Wilson loops in particular backgrounds, they can be used to average Wilson loops over ensembles of Yang-Mills configurations or over different metrics, and in more general settings, see. e.g. [8, 9, 0, 10].

The main aim of this paper is to present new formulae for the gravitational holonomies in curved $d=2,3,4$ spaces: they are similar to our non-Abelian Stokes theorem for the Yang-Mills case. We get rid of the path ordering in eq. (2) and write down the holonomies as exponents of surface integrals. Instead of path ordering we have to integrate over certain covariantly unit vectors (in case of $d=3$ ) or covariantly unit (anti)self-dual tensors (in case of $d=4$ ). Remarkably, these formulae put parallel transporters of different spins on the same footing. In particular, holonomies for half-integer spins are presented in terms of the metric tensor (and its derivatives) only but not in terms of the vielbein or spin connection. 
Apart from purely theoretical interest we have a practical motivation in mind. Quite recently we have shown, both in the continuum and on the lattice, that the $S U(2)$ YangMills partition function in $d=3$ can be exactly rewritten in terms of local gauge-invariant quantities being the six components of the metric tensor of the dual space [11]. This rewriting may be useful to investigate the spectrum and the correlation functions of the theory directly in a gauge-invariant way, but it is insufficient to study the interactions of external sources since they couple to the Yang-Mills potential and not to gauge-invariant quantities. The present paper demonstrates, however, that a typical source, i.e. the Yang-Mills Wilson loop can be expressed not only through the potential (or connection) but also through the metric tensor which is gauge-invariant. Thus, not only the partition function but also Wilson loops in the $d=3$ Yang-Mills theory can be expressed through local gauge-invariant quantities. We leave a detailed formulation of the resulting theory for a forthcoming publication.

Though the main content of the paper are the non-Abelian Stokes theorems for holonomies in 3 and 4 dimensions, we have added three short sections with relevant material. We add for completeness the Stokes theorem in two dimensions, compute the holonomy in a special case of constant curvature with cylinder topology in three dimensions and give a general formula for the 'gravitational Wilson loop' for small loops in any number of dimensions.

\section{Non-Abelian Stokes theorem in Yang-Mills theory}

Let $\tau$ parametrize the loop defined by the trajectory $x^{\mu}(\tau)$ and $A(\tau)$ be the tangent component of the Yang-Mills field along the loop in the fundamental representation of the gauge group, $A(\tau)=A_{\mu}^{a} t^{a} d x^{\mu} / d \tau, \operatorname{Tr}\left(t^{a} t^{b}\right)=\frac{1}{2} \delta^{a b}$. The gauge transformation of $A(\tau)$ is

$$
A(\tau) \rightarrow S(\tau) A(\tau) S^{-1}(\tau)+i S(\tau) \frac{d}{d \tau} S^{-1}(\tau)
$$

Let $H_{i}$ be the generators from the Cartan subalgebra $(i=1, \ldots, r ; r$ is the rank of the gauge group) and the $r$-imensional vector $\mathbf{m}$ be the highest weight of the representation $r$ in which the Wilson loop is considered. The formula for the Wilson loop derived in ref. [1] is a path integral over all gauge transformations $S(\tau)$ which should be periodic along the contour:

$$
W_{r}=\int D S(\tau) \exp i \int d \tau \operatorname{Tr}\left[m_{i} H_{i}\left(S A S^{-1}+i S \dot{S}^{-1}\right)\right] .
$$

Let us stress that eq. (4) is manifestly gauge invariant, as is the Wilson loop itself. For example, in the simple case of the $S U(2)$ group eq. (四) reads:

$$
W_{J}=\int D S(\tau) \exp i J \int d \tau \operatorname{Tr}\left[\tau_{3}\left(S A S^{\dagger}+i S \dot{S}^{\dagger}\right)\right]
$$

where $\tau_{3}$ is the third Pauli matrix and $J=\frac{1}{2}, 1, \frac{3}{2}, \ldots$ is the 'spin' of the representation of the Wilson loop considered.

The path integrals over all gauge rotations (4.5) are not of the Feynman type: they do not contain terms quadratic in the derivatives in $\tau$. Therefore, a certain regularization is understood in these equations, ensuring that $S(\tau)$ is sufficiently smooth. For example, one can introduce quadratic terms in the angular velocities $i S \dot{S}^{\dagger}$ with small coefficients eventually put to zero; see ref. [1] for details. In ref. [1] eq. (5) has been derived in two independent ways: 
i) by direct discretization and ii) by using the standard Feynman representation of path integrals as a sum over all intermediate states, in this case that of an axial top supplemented by a 'Wess-Zumino' type of the action. Another discretization but leading to the same result has been used recently by Kondo [7]. A similar formula has been used by Alekseev, Faddeev and Shatashvili [12] who derived a formula for group characters to which the Wilson loop is reduced in case of a constant $A$ field actually considered in [12]. In ref. [13] eq. (4) has been rederived in an independent way specifically for the fundamental representation of the $S U(N)$ gauge group. Finally, another derivation of a variant of eq. (5) using lattice regularization has been presented recently in ref. [14].

The second term in the exponent of eqs. (4.5) is in fact a 'Wess-Zumino'-type action, and it can be rewritten not as a line but as a surface integral inside a closed contour. Let us consider for simplicity the $S U(2)$ gauge group and parametrize the $S U(2)$ matrix $S$ from eq. (5) by Euler's angles,

$$
\begin{aligned}
S & =\exp \left(i \gamma \tau_{3} / 2\right) \exp \left(i \beta \tau_{2} / 2\right) \exp \left(i \alpha \tau_{3} / 2\right) \\
& =\left(\begin{array}{cc}
\cos \frac{\beta}{2} e^{i \frac{\alpha+\gamma}{2}} & \sin \frac{\beta}{2} e^{-i \frac{\alpha-\gamma}{2}} \\
-\sin \frac{\beta}{2} e^{i \frac{\alpha-\gamma}{2}} & \cos \frac{\beta}{2} e^{-i \frac{\alpha+\gamma}{2}}
\end{array}\right) .
\end{aligned}
$$

The derivation of eq. (5) implies that $S(\tau)$ is a periodic matrix. It means that $\alpha \pm \gamma$ and $\beta$ are periodic functions of $\tau$, modulo $4 \pi$.

The second term in the exponent of eq. (5) which we denote by $\Phi$ is then

$$
\begin{aligned}
\Phi & =\int d \tau \operatorname{Tr}\left(\tau_{3} i S \dot{S}^{\dagger}\right)=\int d \tau \dot{\alpha}(\cos \beta+\dot{\gamma}) \\
& =\int d \tau[\dot{\alpha}(\cos \beta-1)+(\dot{\alpha}+\dot{\gamma})]=\int d \tau \dot{\alpha}(\cos \beta-1) .
\end{aligned}
$$

The last term is a full derivative and can be actually dropped because $\alpha+\gamma$ is $4 \pi$-periodic, therefore even for half-integer representations $J$ it does not contribute to eq. (国). Notice that $\alpha$ can be $2 \pi$-periodic if $\gamma$ (which drops from eq. (7) is $2 \pi, 6 \pi, \ldots$-periodic. If $\alpha(1)=$ $\alpha(0)+2 \pi k, \alpha(\tau)$ makes $k$ windings. Integration over all possible $\alpha(\tau)$ implied in eq. (5) can be divided into distinct sectors with different winding numbers $k$.

Introducing a unit 3-vector

$$
n^{a}=\frac{1}{2} \operatorname{Tr}\left(S \tau^{a} S^{\dagger} \tau_{3}\right)=(\sin \beta \cos \alpha, \sin \beta \sin \alpha, \cos \beta)
$$

we can rewrite $\Phi$ as

$$
\Phi=\frac{1}{2} \int d \tau d \sigma \epsilon^{a b c} \epsilon_{i j} n^{a} \partial_{i} n^{b} \partial_{j} n^{c}, \quad i, j=\tau, \sigma,
$$

where one integrates over any surface spanned on the contour (we shall call it a 'disk'), and $\mathbf{n}$ or $\alpha$ and $\beta$ are continued inside the disk without singularities. We denote the second coordinate by $\sigma$ such that $\sigma=1$ corresponds to the edge of the disk coinciding with the contour and $\sigma=0$ corresponds to the center of the disk. See ref. [14 for details on the continuation inside the disk. 
Let us note that if the surface is closed or infinite the r.h.s. of eq. (9) is the integer topological charge of the $\mathbf{n}$ field on the surface:

$$
Q=\frac{1}{8 \pi} \int d \sigma d \tau \epsilon^{a b c} \epsilon_{i j} n^{a} \partial_{i} n^{b} \partial_{j} n^{c}
$$

Eq. (9) can be also rewritten in a form which is invariant under the reparametrizations of the surface. Introducing the invariant element of a surface,

$$
d^{2} S^{\mu \nu}=d \sigma d \tau\left(\frac{\partial x^{\mu}}{\partial \tau} \frac{\partial x^{\nu}}{\partial \sigma}-\frac{\partial x^{\nu}}{\partial \tau} \frac{\partial x^{\mu}}{\partial \sigma}\right)=\epsilon^{\mu \nu} d(\text { Area })
$$

one can rewrite eq. (9) as

$$
\Phi=\frac{1}{2} \int d^{2} S^{\mu \nu} \epsilon^{a b c} n^{a} \partial_{\mu} n^{b} \partial_{\nu} n^{c}
$$

We get for the Wilson loop [1]:

$$
W_{J}=\int D \mathbf{n}(\sigma, \tau) \exp \left[i J \int d \tau\left(A^{a} n^{a}\right)+\frac{i J}{2} \int d^{2} S^{\mu \nu} \epsilon^{a b c} n^{a} \partial_{\mu} n^{b} \partial_{\nu} n^{c}\right] .
$$

The interpretation of this formula is obvious: the unit vector $\mathbf{n}$ plays the role of the instant direction of the colour 'spin' in colour space; however, multiplying its length by $J$ does not yet guarantee that we deal with a true quantum state from a representation labelled by $J$ - that is achieved only by introducing the 'Wess-Zumino' term in eq. (13): it fixes the representation to which the probe quark of the Wilson loop belongs to be exactly $J$.

Finally, we can rewrite the exponent in eq. (13) so that both terms appear to be surface integrals [2]:

$$
W=\int D \mathbf{n}(\sigma, \tau) \exp \frac{i J}{2} \int d^{2} S^{\mu \nu}\left(-F_{\mu \nu}^{a} n^{a}+\epsilon^{a b c} n^{a}\left(D_{\mu} n\right)^{b}\left(D_{\nu} n\right)^{c}\right)
$$

where $D_{\mu}^{a b}=\partial_{\mu} \delta^{a b}+\epsilon^{a c b} A_{\mu}^{c}$ is the covariant derivative and $F_{\mu \nu}^{a}=\partial_{\mu} A_{\nu}^{a}-\partial_{\nu} A_{\mu}^{a}+\epsilon^{a b c} A_{\mu}^{b} A_{\nu}^{c}$ is the field strength. Indeed, expanding the exponent of eq. (14) in powers of $A_{\mu}$ one observes that the quadratic term cancels out while the linear one is a full derivative reproducing the $A^{a} n^{a}$ term in eq. (13); the zero-order term is the 'Wess-Zumino' term (9) or (7). Note that both terms in eq. (14) are explicitly gauge invariant. We call eq. (14) the non-abelian Stokes theorem. We stress that it is different from previously suggested Stokes-like representations of the Wilson loop, based on ordering of elementary surfaces inside the loop [3, 4, 5, 6]. For a further discussion of eq. (14) see [14].

Let us briefly discuss gauge groups higher than $S U(2)$ : for that purpose we have to return to our eq. (四). Eq. (4) is valid for any group and any representation. It is easy to present it also in a surface form, see ref. [14]. Actually, eq. (4) depends not on all parameters of the gauge transformation but only on those which do not commute with the Cartan combination $\mathcal{H}_{r}=m_{i} H_{i}$. In the $S U(2)$ case one has $m_{i} H_{i}=J \tau_{3}, J=1 / 2,1,3 / 2, \ldots$, since $S U(2)$ is of rank 1 , and there is only one Cartan generator. Therefore, in the $S U(2)$ case one integrates over the coset $S U(2) / U(1)$ for any representation; this coset can be parametrized by the $\mathbf{n}$ field as described above. 
In case of higher groups the particular coset depends on the representation of the Wilson loop. For example, in case the Wilson loop is considered in the fundamental representation of the $S U(N)$ group the combination $m_{i} H_{i}$ is proportional to one particular generator of the Cartan subalgebra, which commutes with the $S U(N-1) \times U(1)$ subgroup. [In case of $S U(3)$ this generator is the Gell-Mann $\lambda_{8}$ matrix or a permutation of its elements.] Therefore, the appropriate coset for the fundamental representation of the $S U(N)$ group is $S U(N) / S U(N-$ 1) $/ U(1)=C P^{N-1}$. A possible parametrization of this coset is given by a complex $N$-vector $u^{\alpha}$ of unit length, $u_{\alpha}^{\dagger} u^{\alpha}=1$. To be concrete, the Cartan combination in the fundamental representation can be always set to be $m_{i} H_{i}=\operatorname{diag}(1,0, \ldots, 0)$ by rotating the axes and subtracting the unit matrix. In such a basis $u^{\alpha}$ is just the first column of the unitary matrix $S$ while $u_{\alpha}^{\dagger}$ is the first row of $S^{\dagger}$. Unitarity of $S$ implies that $u_{\alpha}^{\dagger} u^{\alpha}=1$.

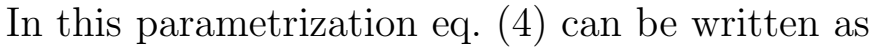

$W_{\text {fund }}^{S U(N)}=\int D u D u^{\dagger} \delta\left(u_{\alpha}^{\dagger} u^{\alpha}-1\right) \exp i \int d \tau \frac{d x^{\mu}}{d \tau} u_{\alpha}^{\dagger}\left(i \nabla_{\mu}\right)_{\beta}^{\alpha} u^{\beta}, \quad\left(\nabla_{\mu}\right)_{\beta}^{\alpha}=\partial_{\mu} \delta_{\beta}^{\alpha}-i A_{\mu}^{a}\left(t^{a}\right)_{\beta}^{\alpha}$.

Using the identity,

$$
\begin{aligned}
\epsilon_{i j} \partial_{i}\left(u^{\dagger} \nabla_{i} u\right) & =\epsilon_{i j}\left[\left(\nabla_{i} u\right)^{\dagger}\left(\nabla_{j} u\right)+u^{\dagger} \nabla_{i} \nabla_{j} u\right] \\
& =\epsilon_{i j}\left[-\frac{i}{2}\left(u^{\dagger} F_{i j} u\right)+\left(\nabla_{i} u\right)^{\dagger}\left(\nabla_{j} u\right)\right],
\end{aligned}
$$

we can present eq. (15) in a surface form:

$$
W_{\text {fund }}^{S U(N)}=\int D u D u^{\dagger} \delta\left(|u|^{2}-1\right) \exp i \int d S^{\mu \nu}\left[\frac{1}{2}\left(u^{\dagger} F_{\mu \nu} u\right)+i\left(\nabla_{\mu} u\right)^{\dagger}\left(\nabla_{\nu} u\right)\right]
$$

where $F_{\mu \nu}$ is the field strength in the fundamental representation. Eq. (17) has been first published in ref. 13 however with an unexpected overall coefficient 2 in the exponent. Eq. (17) presents the non-Abelian Stokes theorem for the Wilson loop in the fundamental representation of $S U(N)$. In the particular case of the $S U(2)$ group transition to eq. (14) is achieved by identifying the unit 3-vector: $n^{a}=u_{\alpha}^{\dagger}\left(\tau^{a}\right)_{\beta}^{\alpha} u^{\beta}$ where

$$
u^{\alpha}=\left(\begin{array}{c}
\cos \frac{\beta}{2} e^{-i \frac{\alpha+\gamma}{2}} \\
\sin \frac{\beta}{2} e^{i \frac{\alpha-\gamma}{2}}
\end{array}\right), \quad 2 i u^{\dagger} \partial_{\tau} u=\dot{\alpha}(\cos \beta-1)+(\dot{\alpha}+\dot{\gamma}) .
$$

It should be mentioned that the quantity

$$
\int d \sigma d \tau \epsilon_{i j} i \partial_{i} u_{\alpha}^{\dagger} \partial_{j} u^{\alpha}=2 \pi Q
$$

appearing in eq. (17) is the topological charge of the 2-dimensional $C P^{N-1}$ model. For closed or infinite surfaces $Q$ is an integer.

In case the Wilson loop is taken in the adjoint represention of the $S U(N)$ gauge group the combination $m_{i} H_{i}$ is the highest root. Only group elements of the form $\exp \left(i \alpha_{i} H_{i}\right)$ commute with this combination, belonging to the maximal torus subgroup $U(1)^{N-1}$. Hence, in case of the adjoint representation one in fact integrates over the maximal coset $S U(N) / U(1)^{N-1}=$ $F^{N-1}$, i.e. over flag variables [15, 7]. 


\section{3 'Gravitational Wilson loops'}

An object similar to the Wilson loop of the Yang-Mills theory exists also in gravity theory. It is the parallel transporter of a vector on a Riemannian manifold along a closed contour, else called a holonomy. The holonomy is trivial if the space is flat but becomes a non-trivial functional of the curvature in case it is nonzero. In the remaining sections we shall present new formulae for the parallel transporters on $d=2,3,4$ Riemannian manifolds.

Let us first remind notations from differential geometry. We use [16] as a general reference book.

Let $g_{\mu \nu}=g_{\nu \mu}(\mu, \nu=1, \ldots d)$ be the covariant metric tensor, with the contravariant $g^{\mu \nu}$ being its inverse, $g_{\mu \nu} g^{\nu \kappa}=\delta_{\mu}^{\kappa}$. The determinant of the covariant metric tensor is denoted by $g$. The Christoffel symbol is defined as

$$
\Gamma_{\nu \kappa}^{\mu}=g^{\mu \lambda} \Gamma_{\lambda, \nu \kappa}=\frac{g^{\mu \lambda}}{2}\left(\partial_{\nu} g_{\lambda \kappa}+\partial_{\kappa} g_{\lambda \nu}-\partial_{\lambda} g_{\nu \kappa}\right), \quad \Gamma_{\nu \kappa}^{\kappa}=\frac{\partial_{\nu} g}{2 g} .
$$

The action of the covariant derivative on the contravariant vector is defined as

$$
\left(\nabla_{\rho}\right)_{\lambda}^{\kappa} v^{\lambda}=\left(\partial_{\rho} \delta_{\lambda}^{\kappa}+\Gamma_{\rho \lambda}^{\kappa}\right) v^{\lambda} .
$$

The commutator of two covariant derivatives determine the Riemann tensor:

$$
\left[\nabla_{\rho} \nabla_{\sigma}\right]_{\lambda}^{\kappa}=R_{\lambda \rho \sigma}^{\kappa}=g^{\kappa \kappa^{\prime}} R_{\kappa^{\prime} \lambda \rho \sigma}=\partial_{\rho} \Gamma_{\sigma \lambda}^{\kappa}-\partial_{\sigma} \Gamma_{\rho \lambda}^{\kappa}+\Gamma_{\rho \tau}^{\kappa} \Gamma_{\sigma \lambda}^{\tau}-\Gamma_{\sigma \tau}^{\kappa} \Gamma_{\rho \lambda}^{\tau} .
$$

A contraction of the Riemann tensor gives the symmetric Ricci tensor,

$$
R_{\lambda \sigma}=R_{\lambda \kappa \sigma}^{\kappa}, \quad R_{\rho}^{\kappa}=R_{\lambda \rho \sigma}^{\kappa} g^{\lambda \sigma} .
$$

Its full contraction is the scalar curvature:

$$
R=R_{\lambda \sigma} g^{\lambda \sigma}=R_{\kappa}^{\kappa} .
$$

The parallel transporter of a contravariant vector along a curve $x^{\mu}(\tau)$ is determined by solving the equation,

$$
\frac{d x^{\mu}}{d \tau}\left(\nabla_{\mu}\right)_{\lambda}^{\kappa} v^{\lambda}(\tau)=0,
$$

whose solution can be written with the help of the evolution operator,

$$
v^{\kappa}(\tau)=\left[W^{G}(\tau)\right]_{\lambda}^{\kappa} v^{\lambda}(0)
$$

where $v^{\lambda}(0)$ is the vector at the starting point of the contour and $v^{\lambda}(\tau)$ is the paralleltransported vector at the point labelled by $\tau$. The evolution operator can be symbolically written as a path-ordered exponent of the Christoffel symbol:

$$
\left[W^{G}(\tau)\right]_{\lambda}^{\kappa}=\left[\mathrm{P} \exp -\int_{0}^{\tau} d \tau \frac{d x^{\mu}}{d \tau} \Gamma_{\mu}\right]_{\lambda}^{\kappa} .
$$

We define the 'gravitational Wilson loop' as the trace of the parallel transporting evolution operator along the closed curve $x^{\mu}(\tau)$ with $x^{\mu}(1)=x^{\mu}(0)$ : 


$$
W_{\text {vector }}^{G}=\frac{1}{d}\left[W^{G}(1)\right]_{\kappa}^{\kappa}
$$

This quantity is diffeomorphism-invariant: if one changes the coordinates $x^{\mu} \rightarrow x^{\prime \mu}(x)$ the metric is transformed, but if one changes the contour accordingly, that is $x^{\mu}(\tau) \rightarrow$ $x^{\prime \mu}(x(\tau))$ the gravitational Wilson loop or the holonomy remains the same. In this respect the gravitational holonomy is different from the Yang-Mills Wilson loop which is invariant under gauge transformation, without changing the contour.

The parallel transporter of a covariant vector is given by the transposed matrix; its trace coincides with that of the contravariant vector.

\section{Relation of gravity quantities to those of the Yang- Mills theory}

We shall show now that the 'gravitational Wilson loop' is not just analogous but directly expressible through the Yang-Mills Wilson loops of the $S U(2)$ group. To that end we introduce the standard vielbein $e_{\mu}^{A}$ and its inverse $e^{A \mu}$ such that

$$
e_{\mu}^{A} e_{\nu}^{A}=g_{\mu \nu}, \quad e_{\mu}^{A} e^{B \mu}=\delta^{A B}, \quad e^{A \mu} e^{A \nu}=g^{\mu \nu}, \quad \operatorname{det} e_{\mu}^{A}=\sqrt{g} .
$$

Let us decompose the vector experiencing the parallel transport in vielbeins,

$$
v^{\lambda}=c^{A} e^{A \lambda}, \quad \text { the reciprocal being } c^{A}=e_{\kappa}^{A} v^{\kappa},
$$

and put it into eq. 25) defining the parallel transport. We have

$$
0=\frac{d x^{\mu}}{d \tau}\left(\nabla_{\mu}\right)_{\lambda}^{\kappa} c^{A} e^{A \lambda}=\frac{d x^{\mu}}{d \tau}\left[e^{A \kappa} \partial_{\mu} c^{A}+c^{A}\left(\partial_{\mu} e^{A \kappa}+\Gamma_{\mu \lambda}^{\kappa} e^{A \lambda}\right)\right]=\frac{d x^{\mu}}{d \tau} e^{B \kappa}\left(\partial_{\mu} \delta^{B A}+\omega_{\mu}^{B A}\right) c^{A}
$$

where we have introduced the spin connection,

$$
\omega_{\mu}^{A B}=-\omega_{\mu}^{B A}=\frac{1}{2} e^{A \kappa}\left(\partial_{\mu} e_{\kappa}^{B}-\partial_{\kappa} e_{\mu}^{B}\right)-\frac{1}{2} e^{B \kappa}\left(\partial_{\mu} e_{\kappa}^{A}-\partial_{\kappa} e_{\mu}^{A}\right)-\frac{1}{2} e^{A \kappa} e^{B \lambda} e_{\mu}^{C}\left(\partial_{\kappa} e_{\lambda}^{C}-\partial_{\lambda} e_{\kappa}^{C}\right),
$$

and used the fundamental relation:

$$
\begin{gathered}
\partial_{\mu} e^{A \kappa}+\Gamma_{\mu \lambda}^{\kappa} e^{A \lambda}=-\omega_{\mu}^{A B} e^{B \kappa} \\
\partial_{\mu} e_{\kappa}^{A}-\Gamma_{\mu \kappa}^{\lambda} e_{\lambda}^{A}=-\omega_{\mu}^{A B} e_{\kappa}^{B} .
\end{gathered}
$$

One can introduce the $S O(d)$ 'field strength',

$$
\mathcal{F}_{\mu \nu}^{A B}=\left[\partial_{\mu}+\omega_{\mu}, \partial_{\nu}+\omega_{\nu}\right]^{A B}=\partial_{\mu} \omega_{\nu}^{A B}-\partial_{\nu} \omega_{\mu}^{A B}+\omega_{\mu}^{A C} \omega_{\nu}^{C B}-\omega_{\nu}^{A C} \omega_{\mu}^{C B},
$$

related to the Riemann tensor as

$$
\mathcal{F}_{\mu \nu}^{A B} e_{\kappa}^{A} e_{\lambda}^{B}=-R_{\kappa \lambda \mu \nu}, \quad \mathcal{F}_{\mu \nu}^{A B}=-R_{\kappa \lambda \mu \nu} e^{A \kappa} e^{B \lambda}, \quad \mathcal{F}_{\mu \nu}^{A B} e^{A \mu} e^{B \nu}=R
$$


The above material is common for any number of dimensions. To proceed further we need to consider separately the cases $d=3$ and $d=4$. The case $d=2$ is considered in section 6 .

\section{$4.1 \mathrm{~d}=3$}

In three dimensions one can immediately identify the spin connection with a Yang-Mills field in $s u(2)$,

$$
A_{i}^{c}=-\frac{1}{2} \epsilon^{a b c} \omega_{i}^{a b}
$$

Speaking about three dimensions we shall denote Lorentz indices by $i, j, \ldots=1,2,3$ and the flat triade indices by $a, b, \ldots=1,2,3$. Recalling the generators in the $J=1$ representation,

$$
\left(T^{c}\right)^{a b}=-i \epsilon^{c a b}, \quad\left[T^{c} T^{d}\right]=i \epsilon^{c d f} T^{f},
$$

we can rewrite the last parenthesis in eq. (31) as

$$
\partial_{i} \delta^{a b}+\omega_{i}^{a b}=\partial_{i} \delta^{a b}-i A_{i}^{c}\left(T^{c}\right)^{a b} \equiv\left(D_{i}\right)^{a b},
$$

which is the standard Yang-Mills covariant derivative in the adjoint representation. In the fundamental (spinor) representation the Yang-Mills covariant derivative is

$$
\left(\nabla_{i}\right)_{\beta}^{\alpha}=\partial_{i} \delta_{\beta}^{\alpha}-i A_{i}^{c}\left(\frac{\sigma^{c}}{2}\right)_{\beta}^{\alpha}=\partial_{i} \delta_{\beta}^{\alpha}+\frac{1}{8} \omega_{i}^{a b}\left[\sigma^{a} \sigma^{b}\right]_{\beta}^{\alpha}, \quad \alpha, \beta=1,2,
$$

which coincides with the known expression for the covariant derivative in the spinor representation in curved space.

The standard Yang-Mills field strength is directly related to that of eq. (35):

$$
F_{i j}^{a}=\partial_{i} A_{j}^{a}-\partial_{j} A_{i}^{a}+\epsilon^{a b c} A_{i}^{b} A_{j}^{c}=-\frac{1}{2} \epsilon^{a b c} \mathcal{F}_{i j}^{b c}
$$

Hence from eq. (36) one has

$$
\epsilon^{a b c} F_{i j}^{a} e_{k}^{b} e_{l}^{c}=R_{i j k l} .
$$

Let us consider the parallel transporter of a 3-vector in curved space, as defined by eq. (25). According to eqs. (31, 39) solving eq. (25) is equivalent to solving the Yang-Mills equation for the parallel transporter,

$$
\frac{d x^{i}}{d \tau}\left(D_{i}\right)^{a b} c^{b}=0
$$

whose solution is

$$
c^{a}(\tau)=\left[W_{1}^{Y M}(\tau)\right]^{a b} c^{b}(0), \quad\left[W_{1}^{Y M}(\tau)\right]^{a b}=\left[\mathrm{P} \exp i \int d \tau \frac{d x^{i}}{d \tau} A_{i}^{c} T^{c}\right]^{a b}
$$

where the subscript " 1 " refers to that the path-ordered exponent is taken in the $J=1$ representation. The parallel transport of a contravariant vector is therefore 


$$
v^{k}(\tau)=c^{a}(\tau) e^{a k}(\tau)=e^{a k}(\tau)\left[W_{1}^{Y M}(\tau)\right]^{a b} e_{l}^{b}(0) v^{l}(0),
$$

from where we immediately get the needed relation between the 'gravitational' and YangMills parallel transporters:

$$
\left[W_{1}^{G}(\tau)\right]_{l}^{k}=e_{k}^{a}(\tau)\left[W_{1}^{Y M}(\tau)\right]^{a b} e^{b l}(0) .
$$

The relation becomes especially neat for the Wilson loops, i.e. for the traces of parallel transporters along closed contours. Since for the closed contour the vielbeins at the end points are the same, $e_{k}^{a}(1)=e_{k}^{a}(0)$, we get

$$
W_{\text {vector }}^{G}=\frac{1}{3}\left[W_{1}^{G}\right]_{k}^{k}=\frac{1}{3}\left[W_{1}^{Y M}\right]^{a a}=W_{1}^{Y M} .
$$

In a similar way one can show that the same equation holds true for the gravitational parallel transporter of covariant vectors and, more generally, for parallel transporters of any integer spin $J$. In this case the Yang-Mills Wilson loop should be taken in the same representation as the gravitational one:

$$
W_{J}^{G}=W_{J}^{Y M} .
$$

In eq. (48) it is understood that the r.h.s. is expressed through the Yang-Mills field equal to the spin connection according to eq. (37), while the l.h.s. is expressed through the Christoffel symbols, that is through the metric. It should be stressed that the spin connection is defined via the vielbein which is not uniquely determined by the metric tensor. Nevertheless, the Wilson loop, being a gauge-invariant quantity, is uniquely determined by the metric tensor and its derivatives. This is the meaning of eq. (48).

For half-integer $J$ there is no way to define the parallel transporter other than through the spin connection. Nevertheless, as we show in section 8 where we present the holonomy for any spin in a surface form, the 'gravitational Wilson loop' is also expressible through the metric tensor and its derivatives, even for half-integer spins.

\section{$4.2 \mathrm{~d}=4$}

In four Euclidean dimensions the rotational group is $S O(4)=S U(2) \times S U(2)$, therefore all irreducible representations of $S O(4)$ can be classified as $\left(J_{1}, J_{2}\right)$ where $J_{1,2}=0, \frac{1}{2}, 1, \ldots$ are the representations of the two $S U(2)$ subgroups. For example, the 4-vector representation whose parallel transporter has been considered in the beginning of this section, transforms as the $\left(\frac{1}{2}, \frac{1}{2}\right)$ representation of $S U(2) \times S U(2)$.

Because of this, it is convenient to decompose the spin connection $\omega_{\mu}^{A B}$ into self-dual and anti-self-dual parts using 't Hooft's $\eta$ and $\bar{\eta}$ symbols. They are defined as

$$
\begin{aligned}
& \eta^{a A B}=\frac{1}{2 i} \operatorname{Tr} \sigma^{a}\left(\sigma^{A+} \sigma^{B-}-\sigma^{B+} \sigma^{A-}\right), \quad \sigma^{A \pm}=( \pm i \sigma, 1), \\
& \bar{\eta}^{a A B}=\frac{1}{2 i} \operatorname{Tr} \sigma^{a}\left(\sigma^{A-} \sigma^{B+}-\sigma^{B-} \sigma^{A+}\right) .
\end{aligned}
$$

We use capital Latin characters to denote flat 4-dimensional vierbein indices, $A, B, \ldots=$ $1,2,3,4$, while $a, b, \ldots=1,2,3 ; \sigma^{a}$ are the three Pauli matrices. The spin connection $\omega_{\mu}^{A B}$ 
transforms as a 6-dimensional representation of $S O(4)$, which can be decomposed as a sum $(1,0)+(0,1)$ of adjoint representations of the two $S U(2)$ subgroups. We write:

$$
\omega_{\mu}^{A B}=-\frac{1}{2} \pi_{\mu}^{a} \eta^{a A B}-\frac{1}{2} \rho_{\mu}^{a} \bar{\eta}^{a A B} .
$$

The $S O(4)$ 'field strength' (35) is then decomposed accordingly:

$$
\mathcal{F}_{\mu \nu}^{A B}=-\frac{1}{2} F_{\mu \nu}^{a}(\pi) \eta^{a A B}-\frac{1}{2} F_{\mu \nu}^{a}(\rho) \bar{\eta}^{a A B}
$$

where

$$
\begin{aligned}
& F_{\mu \nu}^{a}(\pi)=\partial_{\mu} \pi_{\nu}^{a}-\partial_{\nu} \pi_{\mu}^{a}+\epsilon^{a b c} \pi_{\mu}^{b} \pi_{\nu}^{c} \\
& F_{\mu \nu}^{a}(\rho)=\partial_{\mu} \rho_{\nu}^{a}-\partial_{\nu} \rho_{\mu}^{a}+\epsilon^{a b c} \rho_{\mu}^{b} \rho_{\nu}^{c}
\end{aligned}
$$

are the usual Yang-Mills field strengths of the $S U(2)$ Yang-Mills potentials $\pi_{\mu}^{a}$ and $\rho_{\mu}^{a}$. It should be stressed that $6 \cdot 4=24$ variables $\omega_{\mu}^{A B}$ equivalent to $2 \cdot 3 \cdot 4=24$ variables $\pi_{\mu}^{a}, \rho_{\mu}^{a}$ are defined by only $4 \cdot 4=16$ tetrades $e_{\mu}^{A}$ via eq. (32), so that not all of them are independent.

Contracting eq. (36) with the $\eta, \bar{\eta}$ symbols we get:

$$
\begin{aligned}
& F_{\mu \nu}^{a}(\pi)=\frac{1}{2} \eta^{a A B} e^{A \kappa} e^{B \lambda} R_{\kappa \lambda \mu \nu}, \\
& F_{\mu \nu}^{a}(\rho)=\frac{1}{2} \bar{\eta}^{a A B} e^{A \kappa} e^{B \lambda} R_{\kappa \lambda \mu \nu} .
\end{aligned}
$$

Let us now return to the parallel transporter of a 4-vector. As shown in the beginning of this section, finding it is equivalent to solving the equation

$$
\frac{d x^{\mu}}{d \tau}\left(\partial_{\mu} \delta^{A B}+\omega_{\mu}^{A B}\right) c^{B}=0
$$

Let us present the 4-vector $c^{A}$ as a combination of two spinors,

$$
c^{A}=\chi_{\alpha}^{\dagger}\left(\sigma^{A+}\right)_{\beta}^{\alpha} \psi^{\beta}, \quad \chi_{\alpha}^{\dagger} \psi^{\beta}=\frac{1}{2} c^{A}\left(\sigma^{A-}\right)_{\alpha}^{\beta}, \quad \alpha, \beta=1,2 .
$$

Putting it into eq. (57) and decomposing $\omega_{\mu}^{A B}$ as in eq. (51) we get:

$$
\frac{d x^{\mu}}{d \tau}\left\{\partial_{\mu}\left[\chi^{\dagger} \sigma^{A+} \psi\right]-\frac{1}{2}\left(\pi_{\mu}^{a} \eta^{a A B}+\rho_{\mu}^{a} \bar{\eta}^{a A B}\right)\left[\chi^{\dagger} \sigma^{B+} \psi\right]\right\}=0 .
$$

Using the definition of the $\eta$-symbols $(49,50)$ it is easy to check that this equation is satisfied provided the spinors $\chi, \psi$ satisfy

$$
\begin{aligned}
& \frac{d x^{\mu}}{d \tau}\left[\partial_{\mu} \delta_{\beta}^{\alpha}-i \pi_{\mu}^{a}\left(\frac{\sigma^{a}}{2}\right)_{\beta}^{\alpha}\right] \chi^{\beta}=0 \text { or } \frac{d x^{\mu}}{d \tau} \chi_{\alpha}^{\dagger}\left[\overleftarrow{\partial}_{\mu} \delta_{\beta}^{\alpha}+i \pi_{\mu}^{a}\left(\frac{\sigma^{a}}{2}\right)_{\beta}^{\alpha}\right]=0 \\
& \frac{d x^{\mu}}{d \tau}\left[\partial_{\mu} \delta_{\beta}^{\alpha}-i \rho_{\mu}^{a}\left(\frac{\sigma^{a}}{2}\right)_{\beta}^{\alpha}\right] \psi^{\beta}=0 .
\end{aligned}
$$


Expressions in square brackets are identical to the Yang-Mills covariant derivatives, the role of the Yang-Mills potentials played by $\pi_{\mu}^{a}$ and $\rho_{\mu}^{a}$, respectively. Eqs. 60, 61) define the YangMills parallel transporters in the fundamental representation. Their solution can be written as evolution operators,

$$
\begin{aligned}
\chi^{\alpha}(\tau) & =\left[W^{\pi}(\tau)\right]_{\gamma}^{\alpha} \chi^{\gamma}(0) \quad \text { or } \quad \chi_{\alpha}^{\dagger}(\tau)=\chi_{\gamma}^{\dagger}(0)\left[W^{\pi \dagger}(\tau)\right]_{\alpha}^{\gamma}, \\
\psi^{\beta}(\tau) & =\left[W^{\rho}(\tau)\right]_{\delta}^{\beta} \psi^{\delta}(0), \\
{\left[W^{\pi}(\tau)\right]_{\gamma}^{\alpha} } & =\left[\mathrm{P} \exp i \int d \tau \frac{d x^{\mu}}{d \tau} \pi_{\mu}^{a} \frac{\sigma^{a}}{2}\right]_{\gamma}^{\alpha}, \\
{\left[W^{\rho}(\tau)\right]_{\gamma}^{\alpha} } & =\left[\mathrm{P} \exp i \int d \tau \frac{d x^{\mu}}{d \tau} \rho_{\mu}^{a} \frac{\sigma^{a}}{2}\right]_{\gamma}^{\alpha} .
\end{aligned}
$$

Returning to the 4 -vector $c^{A}$ (58) we see that its evolution is determined by

$$
\begin{aligned}
c^{A}(\tau) & =\left[W_{\text {vector }}(\tau)\right]^{A B} c^{B}(0), \\
{\left[W_{\text {vector }}(\tau)\right]^{A B} } & =\frac{1}{2} \operatorname{Tr}\left[W^{\pi \dagger}(\tau) \sigma^{A+} W^{\rho}(\tau) \sigma^{B-}\right] .
\end{aligned}
$$

Let us take a closed contour and take the trace of the evolution operator. The 'gravitational Wilson loop' for a 4-vector is then

$$
\begin{aligned}
W_{\left(\frac{1}{2}, \frac{1}{2}\right)}^{G} & =\frac{1}{4} e^{A \kappa}(1)\left[W_{\text {vector }}(1)\right]^{A B} e_{\kappa}^{B}(0)=\frac{1}{4}\left[W_{\text {vector }}(1)\right]^{A A} \\
& =\frac{1}{2} \operatorname{Tr} W^{\pi} \cdot \frac{1}{2} \operatorname{Tr} W^{\rho} .
\end{aligned}
$$

Its generalization to the holonomy in arbitrary representation $\left(J_{1}, J_{2}\right)$ is obvious:

$$
W_{\left(J_{1}, J_{2}\right)}^{G}=W_{J_{1}}^{\pi} \cdot W_{J_{2}}^{\rho}, \quad W_{J}^{\pi, \rho}=\frac{1}{2 J+1} \operatorname{Tr}_{(2 J+1)} W^{\pi, \rho} .
$$

Thus, in curved $d=4$ space the holonomy in the $\left(J_{1}, J_{2}\right)$ representation is equal to a product of two Yang-Mills Wilson loops where the role of Yang-Mills potentials is played by self-dual $\left(\pi_{\mu}^{a}\right)$ and anti-self-dual $\left(\rho_{\mu}^{a}\right)$ parts of the spin connection. In section 9 we show that both $W^{\pi}$ and $W^{\rho}$ can be written in terms of the metric tensor.

\section{$5 \quad$ Small Wilson loops}

For small-area contours the 'gravitational Wilson loop' can be expanded in powers of the

area. The most straightforward way to do it is to use the path-ordered form of $W^{G}$ as given by eq. (27). We take a square contour of size $a \times a$ lying in the 12 plane, and expand the path-ordered exponent in powers of $a$. After some simple algebra we obtain the first nontrivial term of that expansion which happens to be $O\left(a^{4}\right)$ :

$$
W_{\text {vector }}^{G}=\frac{1}{d}\left[W_{\text {vector }}^{G}\right]_{\kappa}^{\kappa}=1+\frac{a^{4}}{d} R_{\lambda 12}^{\kappa} R_{\kappa 12}^{\lambda}=1-\frac{2(\Delta S)^{\mu \nu}(\Delta S)^{\mu^{\prime} \nu^{\prime}}}{4 d} R_{\kappa \lambda \mu \nu} R_{\rho \sigma \mu^{\prime} \nu^{\prime}} g^{\kappa \rho} g^{\lambda \sigma}
$$


where $(\Delta S)^{\mu \nu}$ is the element of the surface lying in the $(\mu \nu)$ plane. Notice that the first correction to the holonomy is negative-definite. It should be stressed that the first-order term in $\Delta S$ is, generally, present in the expansion of the parallel transporter, however it vanishes when one takes the trace owing to the identity $R_{\kappa \mu \nu}^{\kappa} \equiv 0$, so that the expansion for the trace starts from the $(\Delta S)^{2}$ term.

In $d=3$ eq. (69) can be further simplified because the Riemann tensor is directly related to the Ricci tensor:

$$
R_{i j k l}=R_{i k} g_{j l}-R_{i l} g_{j k}+R_{j l} g_{i k}-R_{j k} g_{i l}+\frac{R}{2}\left(g_{i l} g_{j k}-g_{i k} g_{j l}\right) .
$$

Since Riemann tensor is antisymmetric inside both pairs of subscripts we can replace

$$
g^{k m} g^{l n} \rightarrow \frac{1}{2}\left(g^{k m} g^{l n}-g^{k n} g^{l m}\right)=\frac{1}{2 g} \epsilon^{k l i} \epsilon^{m n j} g_{i j}
$$

We introduce the dual element of the surface,

$$
\Delta S^{p q}=\epsilon^{p q r} \Delta S_{r}
$$

and have

$$
\epsilon^{k l i} \epsilon^{p q r} R_{k l p q}=-4\left(R^{i r}-\frac{1}{2} R g^{i r}\right),
$$

which as a matter of fact is the Einstein tensor. For the parallel transporter of arbitrary spin $J$ the factor "2" in the numerator of (69) should be replaced by $J(J+1)$.

Combining all the factors we obtain

$$
W_{J}^{G}=1-\frac{2 J(J+1)}{3 g}\left(R^{i r}-\frac{1}{2} R g^{i r}\right) g_{i j}\left(R^{j s}-\frac{1}{2} R g^{j s}\right) \Delta S_{r} \Delta S_{s} .
$$

This is our final expression for the trace of the parallel transporter of spin $J$ in a curved $d=3$ space, for small loops. Notice that eq. (74) is invariant under diffeomorphisms.

\section{Gravitational Wilson loop in two dimensions}

In curved $d=2$ space the trace of the parallel transporter along a closed loop can be computed exactly for any metric and presented in the form of a 'Stokes theorem'. The result is related to the Gauss-Bonnet theorem and is generally known: we present it here for the sake of completeness.

The key observation is that in two dimensions the spin connection (32) has only one component,

$$
\omega_{i}^{a b}=\epsilon^{a b} \omega_{i}
$$

In this section all indices assume only two values 1,2. According to eq. (31) the parallel transporter of a vector is determined by the equation

$$
\frac{d c^{a}}{d \tau}-\frac{d x^{i}}{d \tau} \omega_{i} \epsilon^{a b} c^{b}=0
$$


whose solution is

$$
c^{a}(\tau)=W^{a b}(\tau) c^{b}(0), \quad W^{a b}(\tau)=\left(\begin{array}{cc}
\cos \gamma(\tau) & \sin \gamma(\tau) \\
-\sin \gamma(\tau) & \cos \gamma(\tau)
\end{array}\right), \quad \gamma(\tau)=\int_{0}^{\tau} d \tau \frac{d x^{i}}{d \tau} \omega_{i}
$$

According to the general theorem of section 6 the gravitational Wilson loop is equal to the Yang-Mills one, and we obtain

$$
W_{1}^{G}=\frac{1}{2} W^{a a}(1)=\cos \Phi
$$

where

$$
\Phi=\gamma(1)=\int_{0}^{1} d \tau \frac{d x^{i}}{d \tau} \omega_{i}=\frac{1}{2} \oint d x^{i} \epsilon_{a b} \omega_{i}^{a b} .
$$

This formula is not fully satisfactory yet since the holonomy is expressed through the spin connection and not through the metric. It can be achieved if we apply the Stokes theorem and write eq. (79) in a surface form. We have

$$
\Phi=\frac{1}{2} \int d S \epsilon_{a b} \epsilon^{i j} \partial_{i} \omega_{j}^{a b}
$$

where $d S$ is the element of the surface spanned on the contour. Introducing the field strength related to the Riemann tensor,

$$
F_{i j}^{a b}=\partial_{i} \omega_{j}^{a b}-\partial_{j} \omega_{j}^{a b}+\omega_{i}^{a c} \omega_{j}^{c b}-\omega_{j}^{a c} \omega_{i}^{c b}=R_{i j}^{k l} e_{k}^{a} e_{l}^{b}, \quad \epsilon_{a b} e_{k}^{a} e_{l}^{b}=\epsilon_{k l} \sqrt{g},
$$

and noticing that in $d=2$ the commutator term is zero, we rewrite eq. (80) as

$$
\Phi=\frac{1}{2} \int d S \sqrt{g} R, \quad W_{1}^{G}=\cos \Phi,
$$

where $R=(1 / 2) \epsilon^{i j} \epsilon_{k l} R_{i j}^{k l}$ is the scalar curvature. It is gratifying that the holonomy is expressed through the Einstein-Hilbert action, known to be a full derivative in two dimensions. Needless to explain, eq. (82) is diffeomorphism-invariant.

In $d=2$ there is essentially only one component of the Riemann tensor,

$$
R_{1212}=\frac{1}{2} R g
$$

(see [16]). Taking it into account it is easy to check that for small areas the expansion of eq. (82) gives the same result as eq. (69) written for small loops.

\section{An example of big loops: constant curvature back- ground in $d=3$}

In three dimensions the Riemann tensor is expressible through the Ricci tensor, see eq. (70). Because of it, diffeomorphism-invariant information about curved spaces is fully contained in the three eigenvalues of the symmetric Ricci tensor, 


$$
R_{j}^{i}=\lambda \delta_{j}^{i},
$$

the scalar curvature being the sum of the three, $R=\lambda_{1}+\lambda_{2}+\lambda_{3}$. For example, the de Sitter $S^{3}$ space corresponds to $\lambda_{1}=\lambda_{2}=\lambda_{3}=R / 3=$ const. In this section we would like to consider another special case of constant curvature, namely the cylinder space $S^{2} \times R$, characterized by $\lambda_{1}=\lambda_{2}=R / 2=$ const,$\quad \lambda_{3}=0$. We shall see that the parallel transporter in such spaces can be computed for any form of the contour and any metric and that the gravitational Wilson loop is given by an elegant formula.

A general metric can be considered as induced by 6 external coordinates $w^{A}\left(x_{1}, x_{2}, x_{3}\right)$ :

$$
g_{i j}=\partial_{i} w^{A} \partial_{j} w^{A}, \quad A=1, \ldots, 6 .
$$

In the special case of the cylinder space $S^{2} \times R$ it is sufficient to use only four external coordinates $w^{a} \quad(a=1,2,3)$ and $w^{4}$, subject to the constraint

$$
\sum_{a=1}^{3}\left(w^{a}\right)^{2}=\frac{2}{R} .
$$

An example of such external coordinates is given by

$$
w^{1,2,3}(x)=\sqrt{\frac{2}{R}} \frac{x^{1,2,3}}{r}, \quad w^{4}(x)=\sqrt{\frac{2}{R}} \ln r,
$$

leading to the metric tensor

$$
g_{i j}=\frac{2}{R} \frac{1}{r^{2}} \delta_{i j}, \quad \sqrt{g}=\left(\frac{2}{R}\right)^{\frac{3}{2}} \frac{1}{r^{3}} .
$$

A simple calculation using formulae from section 3 shows that this metric indeed gives one eigenvalue of the Ricci tensor zero and the other two equal to a constant $R / 2$. Since the eigenvalues of the Ricci tensor are diffeomorphism-invariant, a general change of coordinates, $x^{i} \rightarrow y^{i}(x)$, in eq. (87) result in the same eigenvalues. Therefore, the most general description of the cylinder spaces $S^{2} \times R$ is given by

$$
\begin{aligned}
w^{a}(x) & =\sqrt{\frac{2}{R}} \frac{y^{a}(x)}{|y(x)|}, \quad w^{4}(x)=\sqrt{\frac{2}{R}} \ln |y(x)|, \quad g_{i j}=\frac{2}{R} \frac{\partial_{i} y^{a} \partial_{i} y^{a}}{y^{2}}, \\
\sqrt{g} & =\left(\frac{2}{R}\right)^{\frac{3}{2}} \frac{1}{3 !} \epsilon^{i j k} \epsilon_{a b c} \frac{\partial_{i} y^{a} \partial_{j} y^{b} \partial_{k} y^{c}}{|y|^{3}}=\frac{1}{2} \sqrt{\frac{R}{2}} \epsilon^{i j k} \epsilon_{a b c} \partial_{i} w^{a} \partial_{j} w^{b} w^{c} \partial_{k} w^{4},
\end{aligned}
$$

where $y^{a}(x)$ are three arbitrary functions of coordinates $x^{i}$. Notice that $g_{i j}$ is given by a product of two matrices $M_{i}^{a}=\partial_{i} y^{a} /|y|$ and hence $\sqrt{g}$ is itself a determinant (of the matrix $M)$.

Our aim is to calculate the Wilson loop for any contour in any metric (89) corresponding to the cylinder spaces. We shall make use of the fact that the Wilson loop is diffeomorphisminvariant. If we compute it for a general contour in some metric representing cylinder spaces, the most general case is recovered by diffeomorphisms of both the contour and the metric. We shall start with the specific metric given by eqs. $(87,88)$. 
Given the metric tensor (88), we construct a vielbein corresponding to it. This is, of course, not unique but any choice of the vielbein will suit us. We choose

$$
e_{i}^{a}=\sqrt{\frac{2}{R}} \frac{1}{r} \delta_{i}^{a}, \quad e_{i}^{a} e_{j}^{a}=g_{i j} .
$$

Given the vielbein we construct the spin connection from its definition (32) or the Yang-Mills field and obtain

$$
A_{i}^{a}=-\frac{1}{2} \epsilon^{a b c} \omega_{i}^{b c}=\epsilon^{a i j} \frac{x^{j}}{r^{2}},
$$

which happens to be the field of the $\mathrm{Wu}$-Yang monopole; the scalar curvature $R$ has dropped from the spin connection. According to the theorem of section 4 the gravitational Wilson loop is equal to the Yang-Mills Wilson loop, provided the Yang-Mills potential $A_{i}^{a}$ is the spin connection of the metric under consideration. Therefore, all we have to do is to compute the Wilson loop in the field of the $\mathrm{Wu}$-Yang monopole, but for a general contour.

This task is easily solvable if we make use of another invariance, this time it is gauge invariance of the Wilson loop. It is well known that the $\mathrm{Wu}$-Yang monopole in the hedgehog gauge (92) can be transformed to the string gauge where the potential has only one nonzero component along the third colour axis (plus a Dirac string). In this gauge the Yang-Mills potential is basically abelian so that one has for the Wilson loop in any representation $J$ :

$$
W_{J}^{G}=W_{J}^{Y M}=\frac{1}{2 J+1} \sum_{m=-J}^{J} \exp i m \Phi, \quad \Phi=\oint d x^{i} A_{i}^{3}=\int d S_{i} \frac{x^{i}}{r^{3}} .
$$

In the last equation we have used the normal Stokes theorem for the circulation and also the fact that in the string gauge the magnetic field of the monopole is the Coulomb field of a point charge; $d S_{i}$ is the element of the surface spanned on the contour, and is orthogonal to the surface.

Eq. (93) is the gravitational Wilson loop for arbitrary contours but in a specific metric given by eq. (88). In order to generalize it to a general metric (89) all one needs is to perform the general coordinate transformation of eq. (93). To this end it is convenient to use, instead of $d S_{i}$, its dual $d S^{i j}$ such that $d S_{i}=\epsilon_{i j k} d S^{j k}$. Taking into account that under the general coordinate transformation $x^{i} \rightarrow y^{i}(x)$ the contravariant vector transforms as $V^{i} \rightarrow V^{k} \partial_{k} y^{i}$, and the antisymmetric contravariant tensor transforms as $d S^{i j} \rightarrow d S^{m n} \partial_{m} y^{i} \partial_{n} y^{j}$, we get for the flux in eq. (93):

$$
\Phi=\int d S_{i} \frac{x^{i}}{r^{3}}=\int d S^{i j} \epsilon_{i j k} \frac{x^{k}}{r^{3}} \rightarrow \int d S^{m n} \frac{\epsilon_{i j k} \partial_{m} y^{i} \partial_{n} y^{j} y^{k}}{|y|^{3}} .
$$

This equation takes a more symmetric form if one uses the external coordinates (89):

$$
\Phi=\left(\frac{2}{R}\right)^{\frac{3}{2}} \frac{1}{2} \int d S_{k} \epsilon_{a b c} \epsilon^{i j k} \partial_{i} w^{a} \partial_{j} w^{b} w^{c}, \quad \sum_{a=1}^{3} w^{a 2}=\frac{2}{R} .
$$

Eq. (93) together with eq. (95) is our final result for the gravitational Wilson loop in the cylinder $S^{2} \times R$ space of constant curvature R. Through eq. (89) the Wilson loop implicitly depends on the metric. Let us make a few comments. 
- The parallel transporter should depend on the metric along the contour but not on the surface spanned on the contour as it can be drawn arbitrarily. Despite the surface form of the result it is indeed so due to the fact that

$$
\partial_{k}\left(\epsilon_{a b c} \epsilon^{i j k} \partial_{i} w^{a} \partial_{j} w^{b} w^{c}\right)=0 .
$$

Therefore, the flux in eq. (95) can be presented as a circulation of a certain vector.

- The flux (95) coincides in form with a well-known expression for the winding number of the mapping $S^{2} \mapsto S^{2}$. For a closed or infinite surface the winding number is normalized as

$$
\frac{1}{8 \pi}\left(\frac{2}{R}\right)^{\frac{3}{2}} \int d S_{k} \epsilon_{a b c} \epsilon^{i j k} \partial_{i} w^{a} \partial_{j} w^{b} w^{c}=Q=\text { integer. }
$$

- For small contours eqs. (93,95) reproduce the result of the previous section. To check it, let us rewrite the general small-loop expansion (69) for the concrete metric (87). We find:

$$
R_{k l p q}=\frac{2}{R r^{6}} \epsilon_{k l u} x^{u} \epsilon_{p q v} x^{v}, \quad g^{i j}=\frac{R}{2} r^{2} \delta^{i j} .
$$

Putting it into eq. (69) and then performing a general coordinate transformation $x^{i} \rightarrow$ $y^{i}(x)$ we obtain after simple algebra

$$
W_{J}^{G}=1-\frac{J(J+1)}{6}\left(\frac{\epsilon_{k l u} y^{u} \partial_{i} y^{k} \partial_{j} y^{l} \Delta S^{i j}}{|y|^{3}}\right)^{2}
$$

which coincides exactly with the expansion of eq. (93) in small loop areas $\Delta S$ up to the second order.

\section{Non-Abelian Stokes theorem in $d=3$ gravity}

In section 4.1 we have shown that the gravitational Wilson loop in integer representations $J$ as a functional of the metric is equal to the Yang-Mills Wilson loop as a functional of the Yang-Mills potential, provided one takes this potential equal to the spin connection corresponding to the metric in question, see eq. (37). For holonomies with half-integer $J$ only the representation via spin connection is available. In this section we present the holonomy both for integer and half-integer $J$ in a unified way, by introducing the "nonAbelian Stokes theorem' similar to that for the Yang-Mills Wilson loop. We shall show that, being written down in a surface form, holonomies for both integer and half-integer $J$ are actually expressible through the metric tensor and its derivatives only: the vielbein and spin connection (not uniquely defined by the metric) are, in fact, unnecessary. 
For any representation $J$ we can use our non-Abelian Stokes formula (14) where the Yang-Mills potential is directly related to the spin connection:

$$
\begin{aligned}
W_{J}^{G} & =W_{J}^{Y M}[\text { spin connection }] \\
& =\int D \mathbf{n} \delta\left(\mathbf{n}^{2}-1\right) \exp \frac{i J}{2} \int d^{2} S^{i j}\left[-F_{i j}^{a} n^{a}+\epsilon^{a b c} n^{a}\left(D_{i} n\right)^{b}\left(D_{j} n\right)^{c}\right] .
\end{aligned}
$$

We shall replace the element of the surface by its dual, $d S^{i j}=\epsilon^{i j p} d S_{p}$. Our goal will be to rewrite this representation of the Wilson loop in terms of the metric of the curved $3 d$ space. To this end we first decompose the integration unit vector $\mathbf{n}$ in the dreibein:

$$
n^{a}=m^{i} e_{i}^{a}, \quad n^{a} n^{a}=m^{i} m^{j} e_{i}^{a} e_{j}^{a}=m^{i} m^{j} g_{i j}=1 .
$$

The new 3 -vector $\mathbf{m}$ is a covariantly unit vector. Since the background metric $g_{i j}$ is fixed we just change the integration variables from $\mathbf{n}$ to $\mathbf{m}$, the new integration measure being

$$
\int D \mathbf{n} \delta\left(\mathbf{n}^{2}-1\right) \ldots=\int D \mathbf{m} \sqrt{g} \delta\left(m^{i} m^{j} g_{i j}-1\right) \ldots
$$

We next use the relation (42) of the field strength $F_{i j}^{a}$ computed from the spin connection $A_{i}^{a}=(1 / 2) \epsilon^{a b c} \omega_{i}^{b c}$, to the Riemann tensor. The first term in the exponent of eq. (101) becomes

$$
\text { first term }=-d S_{p} \epsilon^{i j p}\left(-\frac{1}{2}\right) \epsilon^{a b c} m^{n} e_{n}^{a} R_{k i j}^{l} e_{l}^{b} e^{c k}
$$

Using

$$
\epsilon^{a b c} e^{b l} e^{c k}=\frac{1}{\sqrt{g}} \epsilon^{l k m} e_{m}^{a}, \quad \sqrt{g}=\operatorname{det} e_{i}^{a},
$$

eq. (103) can be continued as

$$
\text { first term }=d S_{p} \epsilon^{i j p} \frac{1}{2 \sqrt{g}} R_{i j k l} \epsilon^{k l m} g_{m n} m^{n} .
$$

The combination of the covariant Riemann tensor and two antisymmetric epsilons has been encountered in section 5 : in $d=3$ it gives the Einstein tensor, see eq. (73). We get therefore

$$
\text { first term }=d S_{p} \sqrt{g}\left(R \delta_{n}^{p}-2 R_{n}^{p}\right) m^{n},
$$

where $R_{n}^{p}$ is the Ricci tensor and $R=R_{k}^{k}$ is the scalar curvature.

We now turn to the second term in the exponent of eq. (101) and again use the decomposition (101). We exploit the fundamental relation (33) which can be presented as

$$
D_{j}^{b b^{\prime}} n^{b^{\prime}}=e_{k}^{b}\left(\nabla_{j}\right)_{l}^{k} m^{l}
$$

where $D_{j}^{b b^{\prime}}=\partial_{j} \delta^{b b^{\prime}}+\epsilon^{b c b^{\prime}} A_{j}^{c}$ is the Yang-Mills and $\left(\nabla_{j}\right)_{l}^{k}=\partial_{j} \delta_{l}^{k}+\Gamma_{j l}^{k}$ is the gravitational covariant derivatives. Therefore, the second term is

$$
\begin{aligned}
\text { second term } & =d S_{p} \epsilon^{a b c} e_{k}^{a} e_{l}^{b} e_{n}^{c} \epsilon^{i j p} m^{k}\left(\nabla_{i}\right)_{l^{\prime}}^{l} m^{l^{\prime}}\left(\nabla_{j}\right)_{n^{\prime}}^{n} m^{n^{\prime}} \\
& =d S_{p} \sqrt{g} \epsilon^{i j p} \epsilon_{k l n} m^{k}\left(\nabla_{i} m\right)^{l}\left(\nabla_{j} m\right)^{n} .
\end{aligned}
$$


Gathering eqs. (102,106, 108) together we get finally a non-Abelian Stokes theorem for the gravitational Wilson loop or the trace of the parallel transporter for spin $J$ along a closed contour:

$$
\begin{aligned}
W_{J}^{G} & =\int D \mathbf{m} \sqrt{g} \delta\left(m^{i} m^{j} g_{i j}-1\right) \\
& \times \exp i \frac{J}{2} \int d S_{k} \sqrt{g}\left[\left(R \delta_{p}^{k}-2 R_{p}^{k}\right) m^{p}+\epsilon^{i j k} \epsilon_{p q r} m^{p}\left(\nabla_{i} m\right)^{q}\left(\nabla_{j} m\right)^{r}\right] .
\end{aligned}
$$

Several comments are in order here.

- The holonomy being defined as a path-ordered exponent is expressed here by a simple exponent of a integral over the surface spanned on the closed contour. That is why we call it a 'Stokes theorem'. The price to pay is a functional integration over covariantly unit vector $\mathbf{m}$ defined on the surface.

- Eq. (109) is invariant under diffeomorphisms, in the sense that if one makes a general coordinate transformation $x^{i} \rightarrow x^{\prime}\left(x^{i}\right)$ and changes the surface appropriately, the holonomy remains invariant.

- The parallel transporter depends only on the contour but should not depend on the way one spans a surface on that contour. The surface integral in eq. (109) has the form

$$
\int d S_{k} \sqrt{g} V^{k}
$$

and the condition that it does not depend on the form of the surface is

$$
\partial_{k}\left(\sqrt{g} V^{k}\right)=0
$$

being equivalent to the condition

$$
\left(\nabla_{k}\right)_{l}^{k} V^{l}=0
$$

since $\Gamma_{k l}^{k}=\Gamma_{l k}^{k}=\partial_{l} \ln \sqrt{g}$. The check of eq. (112) is rather lengthy and we relegate it to the Appendix.

- Condition (112) or equivalently (111) being satisfied means that the surface integral can be written as

$$
\int d S_{k} \sqrt{g} V^{k}=\int d S_{k} \epsilon^{i j k} \partial_{j} B_{k}=-\oint d x^{i} B_{i}
$$

proving that it depends only on the contour, as it should be. However, the vector field $B_{i}$ cannot be uniquely determined from the metric tensor and the covariantly unit vector $\mathbf{m}$.

- The following comment is closely related to the previous. Parallel transporters of integer spins $1,2, \ldots$ are defined via Christoffel's $\Gamma$ symbols and hence by the metric tensor, while parallel transporters of half-integer spins $1 / 2,3 / 2, \ldots$ are not: they are 
defined via the spin connection which is not uniquely constructed from the metric. Nevertheless, it should be expected that the holonomy for half-integer spins, i.e. the trace of a parallel transporter along a closed loop, being a diffeomorphism-invariant quantity, should be expressible through the metric only. The above eq. (109) solves this non-trivial problem: only the metric and its derivatives are involved.

The solution is possible only when one presents the holonomy in the form of a surface integral, as in eq. (109). One cannot do it in a contour form as it is not uniquely expressible through the metric. Were that possible, one would be able to write down a parallel transporter in terms of metric along an open contour as well, but that is not so for half-integer spins.

- Eq. (109) solves another long-standing problem, this time in the Yang-Mills theory. In another paper [11] we have shown that the $S U(2)$ Yang-Mills partition function in three dimensions can be exactly rewritten in terms of gauge-invariant quantities which happen to be the six components of the metric tensor of the dual space. The usual argument why such rewriting is not too useful is that external sources couple to the Yang-Mills potential and not to gauge-invariant quantities. However, now we have demonstrated that a typical source, i.e. the Yang-Mills Wilson loop can be expressed not only through the potential but also through the metric tensor which is gaugeinvariant. Thus, not only the partition function but also Wilson loops in the $d=3$ Yang-Mills theory can be expressed through local gauge-invariant quantities.

\section{Non-Abelian Stokes theorem in $d=4$ gravity}

The aim of this section is to present the holonomy $W_{\left(J_{1}, J_{2}\right)}^{G}$ in curved $d=4$ space in the representation $\left(J_{1}, J_{2}\right)$ in terms of the metric tensor and its derivatives. Eq. (68) presents the holonomy in terms of the (anti)self-dual parts of the spin connection not uniquely determined by the metric, which is not satisfactory. In addition, we would like to get rid of the pathordering in the Yang-Mills Wilson loops $W^{\pi, \rho}$ entering eq. (68). Both goals are achieved via the non-Abelian Stokes theorem similar to that of the previous section, which we are going to derive now.

We start by applying the representation (14) to the Yang-Mills Wilson loop $W^{\pi}$ :

$$
W_{J}^{\pi}=\int D \mathbf{n} \delta\left(\mathbf{n}^{2}-1\right) \exp i \frac{J}{2} \int d S^{\mu \nu}\left[-F_{\mu \nu}^{a}(\pi) n^{a}+\epsilon^{a b c} n^{a}\left(D_{\mu}(\pi) n\right)^{b}\left(D_{\nu}(\pi) n\right)^{c}\right]
$$

where $D_{\mu}^{a b}(\pi)=\partial_{\mu} \delta^{a b}+\epsilon^{a c b} \pi_{\mu}^{c}$ is the covariant derivative with respect to the self-dual part of the spin connection and $F_{\mu \nu}^{a}(\pi)$ is the appropriate field strength (53); it is related to the Riemann tensor via eq. (55). Let us introduce an antisymmetric tensor

$$
m^{\kappa \lambda}=\frac{1}{2} n^{a} \eta^{a A B} e^{A \kappa} e^{B \lambda}
$$

The first term in eq. (114) can be written as $-R_{\kappa \lambda \mu \nu} m^{\kappa \lambda}$. The tensor $m^{\kappa \lambda}$ has actually only two independent components. To see this we introduce two covariant projector operators,

$$
P_{\kappa \lambda \mu \nu}^{+}=\frac{1}{4} \eta^{a A B} \eta^{a C D} e_{\kappa}^{A} e_{\lambda}^{B} e_{\mu}^{C} e_{\nu}^{D}=\frac{1}{4}\left(g_{\kappa \mu} g_{\lambda \nu}-g_{\kappa \nu} g_{\lambda \mu}+\sqrt{g} \epsilon_{\kappa \lambda \mu \nu}\right)
$$




$$
P_{\kappa \lambda \mu \nu}^{-}=\frac{1}{4} \bar{\eta}^{a A B} \bar{\eta}^{a C D} e_{\kappa}^{A} e_{\lambda}^{B} e_{\mu}^{C} e_{\nu}^{D}=\frac{1}{4}\left(g_{\kappa \mu} g_{\lambda \nu}-g_{\kappa \nu} g_{\lambda \mu}-\sqrt{g} \epsilon_{\kappa \lambda \mu \nu}\right)
$$

satisfying projector conditions,

$$
\begin{aligned}
P_{\kappa \lambda \mu \nu}^{ \pm} g^{\mu \mu^{\prime}} g^{\nu \nu^{\prime}} P_{\mu^{\prime} \nu^{\prime} \rho \sigma}^{ \pm} & =P_{\kappa \lambda \rho \sigma}^{ \pm}, \\
P_{\kappa \lambda \mu \nu}^{ \pm} g^{\mu \mu^{\prime}} g^{\nu \nu^{\prime}} P_{\mu^{\prime} \nu^{\prime} \rho \sigma}^{\mp} & =0 \\
P_{\kappa \lambda \mu \nu}^{ \pm} g^{\kappa \mu} g^{\lambda \nu} & =3 .
\end{aligned}
$$

$P_{\kappa \lambda \mu \nu}^{ \pm}$are (covariantly) orthogonal projectors, each having 3 zero and 3 nonzero eigenvalues. They project a general antisymmetric tensor into (covariantly) self-dual and anti-self-dual parts, respectively. It is easy to check that the tensor $m^{\kappa \lambda}$ introduced in eq. (115) is self-dual,

$$
P_{\kappa \lambda \mu \nu}^{-} m^{\kappa \lambda}=0
$$

and satisfies the normalization,

$$
m^{\kappa \lambda} m_{\kappa \lambda}=P_{\kappa \lambda \mu \nu}^{+} m^{\kappa \lambda} m^{\mu \nu}=1,
$$

being a consequence of the normalization $\mathbf{n}^{2}=1$. Therefore, $m^{\kappa \lambda}$ has, indeed, only two independent degrees of freedom in a given metric. We change the integration variables in eq. (114) from $\mathbf{n}$ to $m^{\kappa \lambda}$ :

$$
\int D \mathbf{n} \delta\left(\mathbf{n}^{2}-1\right) \ldots=\int D m^{\kappa \lambda} \sqrt{g} \delta\left(P_{\kappa \lambda \mu \nu}^{-} m^{\mu \nu}\right) \delta\left(m^{\kappa \lambda} m_{\kappa \lambda}-1\right) \ldots
$$

Let us now compute the covariant derivative of $m^{\kappa \lambda}$ :

$$
\begin{aligned}
m_{; \mu}^{\kappa \lambda} & =\partial_{\mu} m^{\kappa \lambda}+\Gamma_{\mu \nu}^{\kappa} m^{\nu \lambda}+\Gamma_{\mu \nu}^{\lambda} m^{\kappa \nu} \\
& =\frac{1}{2} \eta^{a A B}\left[\partial_{\mu} n^{a} e^{A \kappa} e^{B \lambda}+n^{a}\left(\partial_{\mu} e^{A \kappa}+\Gamma_{\mu \nu}^{\kappa} e^{A \nu}\right) e^{B \lambda}+n^{a} e^{A \kappa}\left(\partial_{\mu} e^{B \lambda}+\Gamma_{\mu \nu}^{\lambda} e^{B \nu}\right)\right] \\
& =\frac{1}{2} \eta^{a A B}\left[\partial_{\mu} n^{a} e^{A \kappa} e^{B \lambda}-n^{a} \omega_{\mu}^{A C} e^{C \kappa} e^{B \lambda}-n^{a} e^{A \kappa} \omega_{\mu}^{B C} e^{C \lambda}\right]
\end{aligned}
$$

where in the last equation we have used the fundamental relation (33). We now insert the decomposition of the spin connection $\omega_{\mu}^{A B}$ into self-dual and anti-self-dual parts, eq. (51). Using the relations for the $\eta, \bar{\eta}$ symbols,

$$
\begin{aligned}
\eta^{a A B} \eta^{b A C} & =\delta^{a b} \delta^{B C}+\epsilon^{a b c} \eta^{c B C}, \\
\eta^{a A B} \bar{\eta}^{b A C} & =\eta^{a A C} \bar{\eta}^{b A B}
\end{aligned}
$$

it is easy to see that only the self-dual piece of $\omega_{\mu}^{A B}$ survives in eq. (124), giving

$$
m_{; \mu}^{\kappa \lambda}=\frac{1}{2} \eta^{a A B} e^{A \kappa} e^{B \lambda}\left(\partial_{\mu} \delta^{a b}+\epsilon^{a c b} \pi_{\mu}^{c}\right) n^{b}=\frac{1}{2} \eta^{a A B} e^{A \kappa} e^{B \lambda}\left(D_{\mu}(\pi) n\right)^{a} .
$$

In other words, the gravitational covariant derivative of $m^{\kappa \lambda}$ is expressed through the YangMills covariant derivative of the $\mathbf{n}$ field, which is encountered in the second term of eq. (114). 
Using consecutively eqs. (125, 127) we obtain after simple algebra the final expression for $W_{J}^{\pi}$ (114) but this time presented in terms of the metric:

$$
\begin{aligned}
W_{J_{1}}^{\pi} & =\int D m^{\kappa \lambda} \sqrt{g} \delta\left(P_{\kappa \lambda \mu \nu}^{-} m^{\mu \nu}\right) \delta\left(m^{\kappa \lambda} m_{\kappa \lambda}-1\right) \\
& \times \exp i \frac{J_{1}}{2} \int d S^{\mu \nu}\left[-R_{\kappa \lambda \mu \nu} m^{\kappa \lambda}-\frac{1}{2} \sqrt{g} \epsilon_{\kappa \rho \sigma \tau} g_{\lambda \lambda^{\prime}} m^{\kappa \lambda^{\prime}} m_{; \mu}^{\lambda \rho} m_{; \nu}^{\sigma \tau}\right] .
\end{aligned}
$$

Similarly, $W^{\rho}$ is obtained by integrating over anti-self-dual covariantly unit tensors:

$$
\begin{aligned}
W_{J_{2}}^{\rho} & =\int D m^{\kappa \lambda} \sqrt{g} \delta\left(P_{\kappa \lambda \mu \nu}^{+} m^{\mu \nu}\right) \delta\left(m^{\kappa \lambda} m_{\kappa \lambda}-1\right) \\
& \times \exp i \frac{J_{2}}{2} \int d S^{\mu \nu}\left[-R_{\kappa \lambda \mu \nu} m^{\kappa \lambda}+\frac{1}{2} \sqrt{g} \epsilon_{\kappa \rho \sigma \tau} g_{\lambda \lambda^{\prime}} m^{\kappa \lambda^{\prime}} m_{; \mu}^{\lambda \rho} m_{; \nu}^{\sigma \tau}\right] .
\end{aligned}
$$

As derived in section 4.2 , the gravitational holonomy in representation $\left(J_{1}, J_{2}\right)$ is the product of the two,

$$
W_{\left(J_{1}, J_{2}\right)}^{G}=W_{J_{1}}^{\pi} W_{J_{2}}^{\rho}
$$

Eqs.(128, 129) and (130) form the 'non-Abelian Stokes theorem' for the holonomy in curved $d=4$ space. It expresses the holonomy via surface integrals spanned on the contour, and presents it in terms of the metric tensor and its derivatives only, without reference to the spin connection, even for half-integer representations $J_{1}, J_{2}$.

\section{Conclusions}

The main result of this paper are the non-Abelian Stokes theorems for the holonomies: the Yang-Mills Wilson loop (eq. (14)) and the traces of parallel transporters in curved $d=3$ (eq. (109)) and $d=4$ (eqs. (128,129) ) spaces. In all cases the path-ordered exponents of the connections are removed and replaced by ordinary exponents of surface integrals which, however, do not actually depend on the way the surface is spanned on the contour. The price to pay for the removal of path ordering is high: we obtain functional integrals instead. In the simplest case of the $S U(2)$ Yang-Mills theory it is an integral over a unit 3-vector $\mathbf{n}$ 'living' on the surface, in the case of the $d=3$ Riemannian manifold it is an integral over a covariantly unit 3 -vector $\mathbf{m}$, in case of $d=4$ one integrates over (anti)self-dual covariantly unit tensors.

In spite of functional integration we believe that our formulae are aesthetically appealing. As compared to path-ordered exponents they are better suited to averaging over quantum ensembles of Yang-Mills fields or over various metrics. We hope that elegant formulae can be also used in more general settings.

In addition to the general non-Abelian Stokes formulae we have presented holonomy as a surface integral for a specific background, namely for a $d=3$ space of constant curvature with cylinder topology $S^{2} \times R$. The 'gravitational Wilson loop' is given by a formula for the character whose argument is the winding number of external coordinates, see section 8 .

Parallel transporters of integer spins have a dual description: one can define them either as a path-ordered exponent of Christoffel symbols or as a path-ordered exponent of spin 
connection in appropriate representation. In section 4 we have shown that both representations are equivalent. Even though spin connection is not uniquely determined by the metric tensor, this equivalence means that the holonomy written in terms of spin connection can be in fact expressed through the metric only.

For half-integer spins the situation is far less trivial since the only way to define the holonomy is via the spin connection, and it is not at all clear beforehand that it can be uniquely written through the metric tensor and its derivatives. The non-Abelian Stokes theorem of this paper demonstrates that it is indeed so, but only when one presents the holonomy in the surface form which is uniquely determined by the metric. Though the surface integral does not depend on the way one draws the surface and can be actually written as an integral along the contour, the contour form is not uniquely defined by the surface one, and it reflects the ambiguity in determining the spin connection from the metric.

This finding has interesting implication for Yang-Mills theory in three dimensions, which can be identically reformulated as a quantum gravity theory, with the partition function written as a functional integral over the metric tensor of the dual space [11. This metric tensor is local and gauge invariant (in the Yang-Mills sense). However, one might wish to calculate the average of the Wilson loop which, originally, is defined by the Yang-Mills potential, but not by the metric tensor. In the 'quantum gravity' formulation the Yang-Mills Wilson loop becomes a parallel transporter in the gravitational sense. Therefore, it is very important that it, too, is expressible through the gauge-invariant metric tensor, in any representation. Thus, not only the partition function but also the Wilson loop can be presented in terms of local and gauge-invariant quantities. This subject will be presented in more details elsewhere.

One of us (V.P.) thanks NORDITA for kind hospitality and the Russian Foundation for Basic Research for partial support, grant RFBR-00-15-96610.

\section{Appendix. Proof that eq. (109) does not depend on the surface}

The path-integral representation for the 'gravitational Wilson loop' (109) should not depend on the choice of the surface but only on the contour on which the surface is spanned. To prove that it is indeed so, one has to check eq. (112),

$$
\left(\nabla_{k}\right)_{l}^{k} V^{l}=0
$$

where

$$
V^{k}=\left(R \delta_{p}^{k}-2 R_{p}^{k}\right) m^{p}+\epsilon^{i j k} \epsilon_{p q r} m^{p}\left(\nabla_{i} m\right)^{q}\left(\nabla_{j} m\right)^{r}, \quad m^{i} m^{j} g_{i j}=1 .
$$

To simplify notations we denote covariant derivatives by ";" (see [16]). Explicitly, the covariant derivatives of a scalar, vector, tensor are given by

$$
\begin{aligned}
S_{; k} & =\partial_{k} S, \\
V_{; k}^{i} & =\partial_{k} V^{i}+\Gamma_{k l}^{i} V^{l}, \quad V_{i ; k}=\partial_{k} V_{i}-\Gamma_{i k}^{l} V_{l}, \\
T_{; k}^{i j} & =\partial_{k} T^{i j}+\Gamma_{k l}^{i} T^{l j}+\Gamma_{k l}^{j} T^{i l}, \quad T_{i j ; k}=\partial_{k} T_{i j}-\Gamma_{i k}^{l} T_{l j}-\Gamma_{j k}^{l} T_{i l}, \quad \text { etc. }
\end{aligned}
$$


An ordinary derivative of a convolution of two tensors can be written as a sum of covariant derivatives:

$$
\partial_{k}\left(T_{\ldots}^{(1) \ldots i} T_{\ldots i}^{(2) \ldots}\right)=T_{\ldots ; k}^{(1) \ldots i} T_{\ldots i}^{(2) \ldots}+T_{\ldots}^{(1) \ldots i} T_{\ldots i ; k}^{(2) \ldots}
$$

We apply the covariant derivative to the first term of the vector $V^{k}$ :

$$
\nabla_{k}\left[\left(R \delta_{p}^{k}-2 R_{p}^{k}\right) m^{p}\right]=\left(R \delta_{p}^{k}-2 R_{p}^{k}\right)_{; k} m^{p}+\left(R \delta_{p}^{k}-2 R_{p}^{k}\right) m_{; k}^{p} .
$$

The covariant derivative of the Einstein tensor is known to be zero ([16], eq.(92.10)). Therefore, only the second term in eq. (135) survives.

Next, we apply the covariant derivative to the second term of the vector $V^{k}$ :

$$
\begin{gathered}
\nabla_{k}\left[\epsilon^{i j k} \epsilon_{p q r} m^{p}\left(\nabla_{i} m\right)^{q}\left(\nabla_{j} m\right)^{r}\right] \\
=\epsilon^{i j k} \epsilon_{p q r}\left(\nabla_{k} m\right)^{p}\left(\nabla_{i} m\right)^{q}\left(\nabla_{j} m\right)^{r}+2 \epsilon^{i j k} \epsilon_{p q r} m^{p}\left(\nabla_{i} m\right)^{q}\left(\nabla_{k} \nabla_{j} m\right)^{r} .
\end{gathered}
$$

The first term here is zero, for the following reasons. We differentiate the condition that $m^{i}$ is a covariantly unit vector,

$$
0=\partial_{k}\left(m^{i} m^{j} g_{i j}\right)=2 g_{i j}\left(\nabla_{k} m\right)^{i} m^{j}=2\left(\nabla_{k} m\right)^{i} m_{i},
$$

since the covariant derivative of the metric tensor is zero. It means that three vectors, $\left(\nabla_{1,2,3} m\right)^{i}$ are not linearly independent as there cannot be three linearly independent vectors orthogonal to some vector (in this case $m_{i}$ ) in three dimensions. The first term in eq. (136) is an antisymmetrized product of these three linearly-dependent vectors, therefore, it is zero.

As to the second term in eq. (136) we notice that it contains the commutator of covariant derivatives, equal to

$$
\epsilon^{i j k}\left(\nabla_{k} \nabla_{j} m\right)^{r}=\frac{1}{2} \epsilon^{i j k}\left[\nabla_{k} \nabla_{j}\right]_{s}^{r} m^{s}=\frac{1}{2} \epsilon^{i j k} g^{r t} R_{t s k j} m^{s}
$$

where $R_{t s k j}$ is the Riemann tensor. Therefore, the second (and the only nonzero) term in eq. (136) can be written as

$$
\epsilon^{i j k} \epsilon_{p q r} g^{r t} R_{t s k j} m^{p} m^{s}\left(\nabla_{i} m\right)^{q} .
$$

We next use eq. (70) to express the Riemann tensor through the Ricci and metric tensors and write down the product of two epsilons as a determinant made of Kronecker deltas. Performing all convolutions we obtain that eq. (139) can be identically rewritten as

$$
\left[g_{q s}\left(R \delta_{p}^{i}-2 R_{p}^{i}\right)-g_{p s}\left(R \delta_{q}^{i}-2 R_{q}^{i}\right)\right] m^{p} m^{s}\left(\nabla_{i} m\right)^{q}
$$

Here the first term is zero because of eq. (137) while in the second term we use $g_{p s} m^{p} m^{s}=1$. As a result we get

$$
-\left(R \delta_{q}^{i}-2 R_{q}^{i}\right)\left(\nabla_{i} m\right)^{q}
$$

which cancells exactly with eq. (135). Thus, $\left(\nabla_{k}\right)_{l}^{k} V^{l}=0$, q.e.d. 


\section{References}

[1] D.Diakonov and V.Petrov, Sov. Phys. JETP Lett. 49 (1989) 284;

Phys. Lett. B224 (1989) 131.

[2] D.Diakonov and V.Petrov, in: Nonperturbative Approaches to Quantum Chromodynamics, Proc. of the International workshop in ECT*, Trento, ed. D.Diakonov, Gatchina (1995) p. 36; hep-th/9606104.

[3] M.B.Halpern, Phys. Rev. D19 (1979) 517.

[4] I.Ya.Aref'eva, Theor. Math. Phys. 43 (1980) 353.

[5] N.Bralic, Phys. Rev. D22 (1980) 3090.

[6] Yu.A.Simonov, Sov. J. Nucl. Phys. 50 (1989) 134.

[7] K.-I.Kondo and Y.Taira, Non-Abelian Stokes Theorem and Quark Confinement in $S U(N)$ Yang-Mills Gauge Theory, hep-th/9911242.

[8] D.Diakonov and V.Petrov, Phys. Lett. 242 (1990) 425.

[9] A.M.Polyakov, Nucl. Phys.Proc.Suppl. 68, 1 (1998), hep-th/9711002.

[10] C.Korthals-Altes and A.Kovner, Magnetic Z(N) Symmetry in Hot QCD and the Spatial Wilson Loop, hep-ph/0004052.

[11] D.Diakonov and V.Petrov, Yang-Mills Theory in Three Dimensions as Quantum Gravity, hep-th/9912268.

[12] A.Alekseev, L.Faddeev and S.Shatashvili, J. Geom. Phys. 5 (1989) 391.

[13] F.A.Lunev, Nucl. Phys. B494 (1997) 433.

[14] D.Diakonov and V.Petrov, On the Non-Abelian Stokes Theorem, hep-lat/0008004.

[15] A.M.Perelomov, Generalized Coherent States and their Applications, Springer Verlag, N.Y. (1986);

Phys. Reports 146 (1987) 135.

[16] L.D.Landau and E.M.Lifshitz, The Classical Theory of Fields, Butterworth-Heinemann (1997). 